\title{
Microvascular damage evaluation in systemic sclerosis: the role of nailfold videocapillaroscopy and laser techniques
}

\author{
B. Ruaro', A. Sulli', V. Smith², C. Pizzorni' ${ }^{1}$, S. Paolino', E. Alessandri', M. Cutolo' \\ ${ }^{1}$ Research Laboratory and Academic Division of Clinical Rheumatology, Department of Internal Medicine, \\ University of Genova, IRCCS San Martino-IST, Genova, Italy; '2Department of Rheumatology, Ghent University \\ Hospital, Department of Internal Medicine, Ghent University, Ghent, Belgium
}

\begin{abstract}
SUMMARY
Microvascular damage and a decrease in peripheral blood perfusion are typical features of systemic sclerosis (SSc) with serious clinical implications, not only for a very early diagnosis, but also for disease progression. Nailfold videocapillaroscopy is a validated and safe imaging technique able to detect peripheral capillary morphology, as well as to classify and to score any nailfold abnormalities into different microangiopathy patterns. Capillaroscopic analysis is now included in the ACR/EULAR classification criteria for SSc.

The decrease in peripheral blood perfusion is usually associated with microvascular damage in SSc, which may be studied by different methods. Several of these make use of safe laser technologies.

This paper focuses on these new clinical aspects to assess SSc microvascular impairment.
\end{abstract}

Key words: Systemic sclerosis; Raynaud phenomenon; Microcirculation; Nailfold videocapillaroscopy; Laser techniques.

\section{INTRODUCION}

icrovascular changes, characterized by structural and functional abnormalities of the microcirculation, play a central role in the pathogenesis of systemic sclerosis $(\mathrm{SSc})$. Nailfold videocapillaroscopy (NVC) represents a validated, noninvasive, safe, inexpensive, reliable and reproducible method for the evaluation of the structural changes that occur in the peripheral microcirculation in SSc (1-4). Since microvascular damage and dysfunction are early markers of SSc and are clinically mirrored by secondary Raynaud's phenomenon (RP), the diagnostic, prognostic and therapeutic implications of microvessel morphological analysis by NVC allows for the best clinical management. $\mathrm{RP}$ is often the earliest clinical symptom of digital microcirculatory damage and can be considered one of the risk factors for the development of a connective tissue disease. NVC detects and quantifies the microvascular changes that characterize secondary $\mathrm{RP}(1,2,5,6)$. It also distinguishes second- ary RP from both primary RP and healthy subjects, identifies morphological patterns specific to various SSc microangiopathy stages (Early, Active and Late patterns) and allows for the calculation of the microangiopathy evolution score (MES) to follow disease evolution (4-8).

Although NVC cannot measure blood perfusion under standard conditions, assessment of blood perfusion in SSc may be performed by different laser techniques (911). The use of NVC in combination with laser equipment provides a morphological and functional assessment of the peripheral microcirculation that is a must for diagnosis, prognosis and therapy in SSc patients.

\section{THE PERFORMANCE OF NAILFOLD VIDEOCAPILLAROSCOPY}

Patients affected by RP represent a diagnostic challenge as there is a broad possibility of differential diagnosis. They represent the main indication for NVC, as the technique distinguishes primary from secondary RP.
Corresponding author: Maurizio Cutolo Research Laboratory and Academic Division of Clinical Rheumatology, Department of Internal Medicine, University of Genova

Viale Benedetto XV, 6 16132 Genova, Italy E-mail: mcutolo@unige.it 
Videocapillaroscopy is a combination of an optical microscope (best lens magnification 200x) and a digital video camera. The patient under investigation should initially remain seated in an acclimatized room for 15-20 min, with a set temperature of about $22-23^{\circ} \mathrm{C}$. A drop of immersion (cedar) oil is placed onto the cuticle of the fingers to be analysed in order to reduce any refractive defects and improve capillary visualization $(3,12)$.

The morphology of nailfold dermal capillaries is assessed by NVC at the level of the periungual region from the $2^{\text {nd }}$ to the $5^{\text {th }}$ finger (excluding the thumb, due to a lower transparency caused by the nail width and the absence of a distal phalanx) of both hands, as the capillaries run parallel to the skin surface in this region $(3,12)$. The qualitative NVC assessment of the capillaries in this region can distinguish a normal pattern from an abnormal one.

Useful capillaroscopic parameters are: capillary density, capillary morphology, number of capillaries inside each dermal papilla, the disposition of the capillaries in the nailfold bed, the occurrence of ramifications and presence of haemorrhages and/ or haemosiderin deposit, skin transparency, visibility and morphology of the subpapillary venous plexus $(3,12)$.

The normal range of nailfold capillaries density is 9-12 capillaries per linear millimetre. The number of capillaries may be slightly less in the early phase of SSc, whilst the progressive capillary desertification is a diagnostic feature of advanced SSc microangiopathy $(2,12)$. In normal subjects the capillaries are parallel to one other in their normal disposition, in the number of one (rarely two) per dermal papilla, and are perpendicularly oriented towards the nailfold. A qualitative assessment of a normal capillaroscopic pattern is characterized by a homogeneous distribution of hairpin-shaped capillaries with a comb-like structure. The capillary normal caliber is usually $<20 \mu \mathrm{m}$.

A capillary is defined as ectasic when dilated at $\geq 20 \mu \mathrm{m}$ (but $<50 \mu \mathrm{m}$ ), whilst a giant capillary is defined as a vessel with a homogeneous dilation exceeding $50 \mu \mathrm{m}$ (involv- ing both afferent and efferent branches). A recent study demonstrated that dilations in a capillary diameter of $>30$ micrometers at the level of arterial and/or venous branches were significantly expressed in those subjects with primary RP that later developed secondary RP, associated with SSc (mean follow-up 3.56 years) (13). Furthermore, the observation of abnormal capillary dilations in primary RP subjects should be taken as a possible very early NVC sign of transition towards the Early scleroderma pattern of microangiopathy (13).

Three different capillary morphologies may be observed in normal capillaries: stereotype hairpin shapes (present in 50\% of healthy subjects); nonspecific variations like tortuosities (40\% of controls); crossing capillaries ( $8 \%$ of controls) (14).

Another study reported that capillary branching $(78 \%)$, meandering capillaries $(94 \%)$, tortuous loops $(64 \%)$, microhaemorrhages $(25 \%)$ and apical dilatations (19\%) may also be found in normal subjects, even if abnormal shapes usually occur with a low prevalence (15). These variations could be influenced by the subjects' profession, trauma, age, onychophagy, manicures, exposure to chemical substances and/or individual physiological variability $(1-3,14)$.

The subpapillary venous plexus visibility and the presence of oedema are considered non-specific findings that are not of univocal interpretation $(1-3,12)$.

Although nailfold capillaries are frequently normal in primary RP, capillaries with efferent branch enlargement or tortuous capillaries may be observed in this clinical situation. Conversely, secondary RP is characterized by morphological signs that represent the microvascular damage, including giant capillaries, microhaemorrhages, capillary loss, the presence of avascular areas and angiogenesis.

Several sequential capillaroscopic changes are typical of the microvascular involvement observed in more than $95 \%$ of SSc patients, and have been described by the term scleroderma-pattern $(1-3,12)$. The NVC technique identifies those capillary patterns that have the morphological features of the 
scleroderma-pattern of microangiopathy. However, these may be observed not only in $\mathrm{SSc}$, but also in mixed connective tissue disease, dermatomyositis and, less frequently, in undifferentiated connective tissue diseases as well as in lupus erythematosus (13,12 ). Indeed, specific capillaroscopic patterns have been described only in systemic sclerosis $(1-3,12,16,17)$.

A recent study by Cutolo et al. on a large cohort demonstrated that almost $16 \%$ of patients initially diagnosed as being affected by RP with negative NVC may transit to secondary RP over an average followup of 4.4 years (16). Primary RP patients with non-specific nailfold capillary alterations (dilations of capillary diameter $>30$ micrometer at the level of arterial branch, microhaemorrhages, reduction of capillaries number $<9$ ) at first NVC evaluation should be closely monitored at least every six months, as they run a higher risk of transition to secondary RP $(2,13,16)$.

\section{THE NAILFOLD VIDEOCAPILLAROSCOPIC SCLERODERMA-PATTERNS}

The first modern interpretation of the microvascular alterations in SSc was given by Maricq et al. in 1980, using the wide-field microscopy technique, describing it as a scleroderma-type capillaroscopic pattern (18). This classification was later revised by Cutolo et al. in 2000, when they introduced a new concept on the basis of the selected microvascular characteristics of disease progression. The microvascular lesions detected by NVC in SSc patients follow three clearly distinct patterns that differ from the normal aspect and reflect various phases of capillary changes. They have an evolutive trend from the first (Early), to the second (Active), until the third (Late) microangiopathy capillaroscopic patterns $(1-3,12,19,20)$. Each one is distinguished by typical and univocal capillaroscopic abnormalities and correlates with the disease duration and autoantibody profile (20-24). The Early NVC scleroderma pattern is the most significant pattern, as it affords an early SSc and scleroderma-spectrum dis- ease diagnosis. It is defined as the combination of a few enlarged/giant capillaries, a few capillary microhaemorrhages, a relatively well preserved capillary distribution and no evident or minimal capillary loss (Figure 1). The Active NVC scleroderma pattern features frequent giant capillaries, frequent capillary microhaemorrhages, a moderate capillary loss, mild disorganization of the capillary architecture and absent or mild ramified capillaries (Figure 1).

Microhaemorrhages may be detected in the Early and Active phase of SSc microangiopathy and are associated with early vascular damage. Indeed, their presence represents a transition phase between giant capillaries and the subsequent loss of capillaries. However, microhaemorrhages are not specific only for the scleroderma spectrum disorders and may be found in other connective tissue diseases, such as systemic lupus erythematosus or antiphospholipid syndrome (for example in antiphospholipid syndrome microhaemorrhages with linear and parallel disposition to capillaries bed are described); furthermore microhaemorrhages may be also post-traumatic and in the case of recent manicure misleading mi-

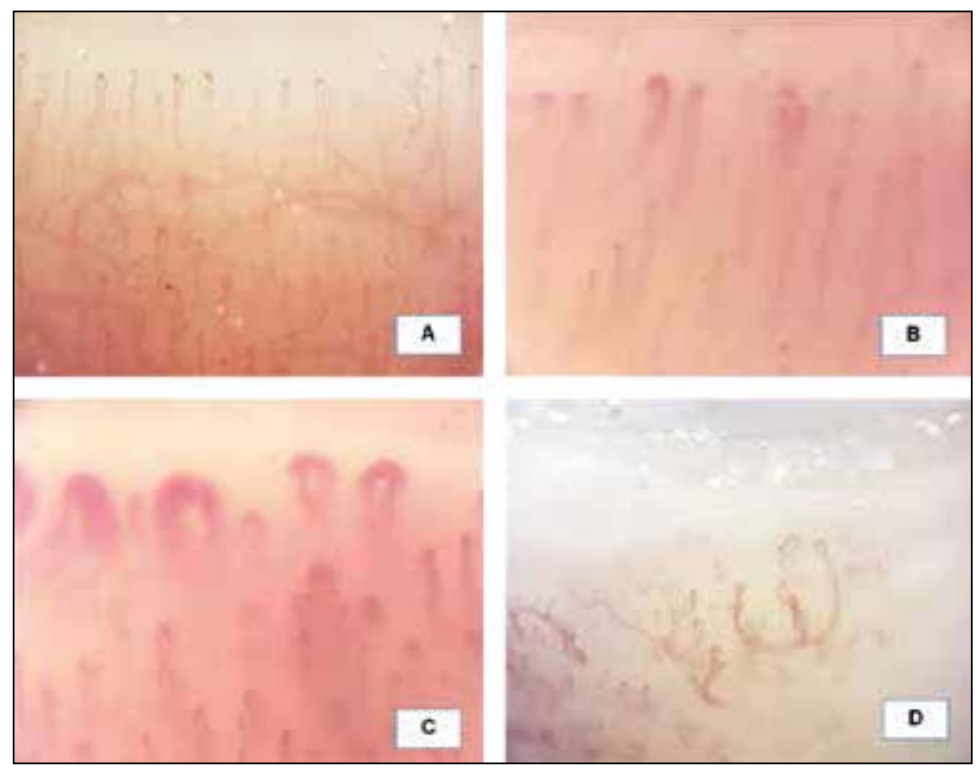

Figure 1 - Nailfold videocapillaroscopy images (x200) in healthy subject (A), Early (B), Active (C) and Late (D) patterns of scleroderma microangiopathy. 
crobleedings may be frequently observed) (1-3, 12).

The Late scleroderma NVC pattern shows an irregular enlargement of the capillaries, only a few or absence of giant capillaries and microhaemorrhages, severe capillary loss with avascular areas, disorganization of the normal capillary array and ramified/ bushy capillaries (Figure 1).

A scleroderma-like pattern may be observed in SSc treated patients (several treatments may change the nailfold capillary bed characteristics, either increasing capillary number and neoangiogenic capillaries or reducing giant capillary and microhaemorrage number), as well as in connective tissue diseases other than SSc. The scleroderma-like pattern is defined as a capillary pattern with a mixture of the microvascular abnormalities included in the scleroderma capillary patterns, but one that does not fully fit the definition of any of the three aforementioned scleroderma patterns $(1-3,12)$.

In 2008, Sulli et al. proposed to assess the NVC parameters belonging to the scleroderma pattern in a quantitative manner (enlarged capillaries, giant capillaries, microhaemorrhages, capillary loss, disorganization of the microvascular array and capillary ramifications) $(4,8)$. To this aim, a semiquantitative rating scale to score these altered microvascular parameters was adopted $(4,8)$. The capillary abnormalities were scored according to their intensity as follows: $0=$ no changes/reduction; $1=<33 \%$ of capillary changes/reduction; $2=33 \%$ to $66 \%$ of capillary changes/reduction; $3=>66 \%$ of capillary changes/reduction. The average score for each capillaroscopic parameter was calculated from the analysis of four consecutive fields (1 linear mm for each one) in the middle of the nailfold bed, on each digit. The average scores from the eight digits were then summed together and the final value was divided for eight fingers. The resulting value represented the score for each capillaroscopic parameter analyzed $(4,8)$. By convention, an abnormal capillaroscopic finding is considered significant if it is observed in at least two of the subject's fingers $(2,3,15)$.
The microangiopathy evolution score (MES), made up of the sum of three parameter scores (capillary loss, disorganization of the microvascular array and capillary ramifications), was proposed to assess the progression of the vascular damage (4, $8)$. An increase in the trend of these three alterations was observed during SSc progression, whilst giant capillaries and microhaemorrhages were not considered to be of critical value in assessing the evolution of the SSc microangiopathy, as these abnormalities are evident only in the early stages of the disease and then either disappear or become rare in the more advanced stages $(2,3)$. The MES showed a statistically significant change throughout the follow-up, confirming its utility in quantifying and monitoring the SSc microvascular damage $(4,8)$.

\section{CLINICAL APPLICATION OF NAILFOLD CAPILLAROSCOPY IN SYSTEMIC SCLEROSIS}

Cross-sectional and predictive associations between progressive capillaroscopic changes (detecting microvascular damage) and clinical applications in SSc have recently been described. One study described the association between progressive changes in capillary morphology and disease subset and severity (23). Other studies that confirm the predictive role of capillaroscopy for digital trophic lesions in SSc have been published $(25,26)$. Literature reports two single centre prospective studies that propose prognostic indices able to predict future progressive vascular disease in SSc patients on the basis of quantitatively assessed hallmark parameters involved in the scleroderma pattern (i.e., capillary loss and/or giant capillaries) $(25,26)$. Smith et al. also demonstrated the role NVC plays in the prediction of both digital ulcers and organ involvement (27).

A recent international study (the CAP study) linking the nailfold capillaroscopic parameters to the progression of digital ulcers in SSc (700 patients, 14 Countries and 59 Centres) has defined NVC analysis as a predictive tool (28). Moreover, Ricceri et 
al. reported that NVC damage correlates with the grade of pulmonary arterial hypertension $(29,30)$.

Interestingly, NVC and related scores may also be used to monitor the effects of the therapies adopted in SSc treatment. However, to date there are only some pilot studies (no prospective, no randomized, no double blind and no placebo controlled) on this issue (31-43).

It has been observed that long term treatment with the endothelin receptor antagonist bosentan, in combination with a vasodilator (iloprost), in SSc patients affected by digital ulcers blocked the evolution of the microvascular damage after one year, as assessed by both NVC and peripheral blood flow analysis (37-40). Furthermore, some studies have made use of NVC to detect the response to immunosuppressive treatment for SSc severity outcome measures (41-43).

All of which led to NVC being included as a parameter in the 2001 LeRoy criteria for the classification of early systemic sclerosis (44). More recently, it was included in the 2013 VEDOSS criteria for very early diagnosis of SSc and in the new 2013 ACR/ EULAR classification criteria for SSc, and in 2014 diagnostic criteria for primary RP (45-47).

The use of capillaroscopy by physicians, in their routine clinical practice, was encouraged also by the authors of the 2013 ACR/ EULAR classification criteria for SSc (46). On the other hand, a recent multicentre international study demonstrated that even the less skilled were able to use NVC to distinguish normal from abnormal capillaries, after only one hour of training (48).

\section{CAPILLAROSCOPY LINKS TO LASER TECHNIQUES FOR THE ANALYSIS OF THE PERIPHERAL BLOOD FLOW}

As previously mentioned, NVC is unable to measure the capillary blood flow/perfusion under standard conditions, since only a morphological evaluation of the vascular array can be performed (8). Therefore, some studies have combined NVC to various other techniques able to assess and quantify cutaneous blood perfusion (numerous laser techniques, thermography and photoplethysmography) (49-56).

Several studies have demonstrated that SSc patients have a lower peripheral blood flow than do both healthy subjects and primary RP patients, and that patients with the Late NVC pattern of microangiopathy have a lower blood flow at laser Doppler flowmetry (LDF) than patients with Active and Early NVC patterns $(37,40)$.

LDF is also efficacious in the evaluation of the variation in peripheral blood perfusion during treatment with vasodilator drugs within a few days, or even during a long follow-up $(37,40)$. However, one limit of the LDF technique is the possible site-tosite variation (when moving the probe to evaluate the perfusion in different areas), limiting its efficacy in comparing blood flow between sites and in monitoring its change over time $(37,40,53)$.

However, laser Doppler imaging (LDI), which evaluates blood flow over a skin area, might well overcome this problem $(49,52)$. Murray et al. reported that NVC, LDI and thermal imaging (a technique that measures indirect blood flow) each independently provide for a good discrimination between patients with SSc and those with primary RP and healthy controls. They observed that the combination of all three techniques improves the classification of SSc patients and that LDI and thermal imaging give equivalent information on dynamic changes in the cutaneous microcirculation. Moreover, they confirmed that NVC is the best method for classifying patient groups (49).

Rosato et al. used LDI and photoplethysmography (which uses infrared light to evaluate any changes in blood volume, and has been shown to have a positive concordance with LDI) to study skin perfusion and digital artery pulsatility of the hands in patients with primary RP and SSc simultaneously. They demonstrated that, when the two tools are used together they are able to provide useful information in distinguishing patients with primary RP from SSc patients, although NVC remains the best 
method for analyzing microvascular damage (52).

The laser speckle contrast analysis (LASCA), which measures skin perfusion using the speckle principle, has the advantage of being able to quantify blood perfusion over a large area and is a non-contact technique $(53,54)$. A recent study demonstrated that LASCA has a very good inter-rater reliability for peripheral blood perfusion measurements (55). Indeed, LASCA has been applied in research studies on RP and SSc $(53,54)$ and one such study demonstrated that peripheral blood perfusion evaluated by both LDF and LASCA correlates with the extent of the microangiopathy (53).

It has also been reported that when patients with the Late SSc microangiopathy pattern were evaluated by both methods, a lower blood flow than that of patients with the Active or Early SSc patterns on NVC, was observed (Figure 2) (53). Another study, which used the LASCA technique for assessment, reported that blood perfusion was significantly lower in SSc patients than in healthy subjects at the level of fingertips, periungual areas and palms of the hands and that there was a statistically significant negative correlation between the extent of the nailfold microangiopathy and the perfusion values at the level of the same skin areas in SSc patients (56).

Another study demonstrated that LASCA
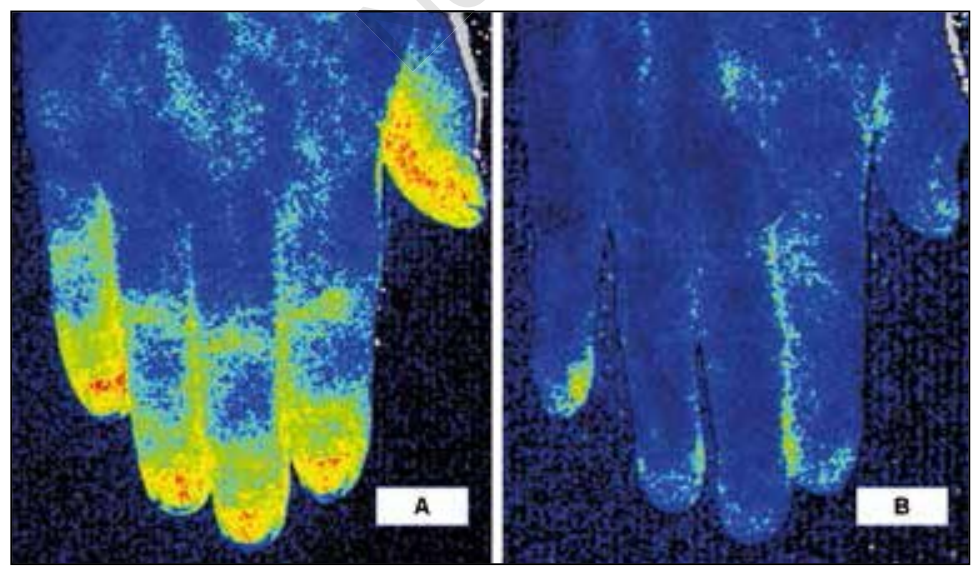

Figure 2 - Laser speckle contrast analysis images of the hand dorsal aspect in a healthy subject $(A)$ compared to a patient with a Late scleroderma pattern of microangiopathy (B). Blue = low blood perfusion; yellow = intermediate blood perfusion; red = high blood perfusion. is able to safely monitor digital ulcer evolution in SSc patients, by evaluating the blood perfusion in different areas (surrounding and within the skin lesions) during standard treatment (57). A recent study has demonstrated that LASCA is also a good tool for evaluating the variation in peripheral blood perfusion during a longterm follow-up (58).

Other techniques, such as thermal imaging, are able to make an indirect evaluation of blood perfusion, as they measure the skin temperature. Thermal imaging has been used in several studies to differentiate between primary RP and SSc. Distal dorsal difference $>1{ }^{\circ} \mathrm{C}$ between one or more fingertips and the dorsum of the hand (fingers cooler) at $+30^{\circ} \mathrm{C}$ seems predictive of a SSc-spectrum disorder (59).

\section{CONCLUSIONS}

There has been a rapid development of new methods with which to study microvascular damage in SSc patients over the last few years and these techniques have the advantage of being non-invasive, safe, reproducible and quantifiable. Currently, NVC is the validated tool able to assess the microvascular damage present in SSc patients in daily practice. Other complementary laser techniques have been used in pilot clinical research studies, demonstrating their usefulness for monitoring the effects of treatments on skin blood perfusion, and need further validation.

Acknowledgments: the authors thank Barbara Wade, contract Professor at the University of Torino, for her linguistic advice. The authors thank Doctor Sara De Gregorio from the Division of Rheumatology, University of Genova, for their support in graphic reproduction. Barbara Ruaro is supported by a Grant from the Italian Society of Rheumatology 2015. All authors are members of the EULAR Study Group on Microcirculation in Rheumatic Diseases.

Conflict of interest: Maurizio Cutolo has received research funds from Actelion, BMS and Sanofi-Aventis. 


\section{REFERENCES}

1. Herrick AL, Cutolo M. Clinical implications from capillaroscopic analysis in patients with Raynaud's phenomenon and systemic sclerosis. Arthritis Rheum. 2010; 62: 2595-604.

2. Cutolo M, Sulli A, Smith V. Assessing microvascular changes in systemic sclerosis diagnosis and management. Nat Rev Rheumatol. 2010; 6: 578-87.

3. Cutolo M, Sulli A, Smith V. How to perform and interpret capillaroscopy. Best Pract Res Clin Rheumatol. 2013; 27: 237-48.

4. Smith V, Pizzorni C, De Keyser F, et al. Reliability of the qualitative and semiquantitative nailfold videocapillaroscopy assessment in a systemic sclerosis cohort: a two-centre study. Ann Rheum Dis. 2010; 69: 1092-6.

5. Cutolo M, Grassi W, Matucci Cerinic M. Raynaud's phenomenon and the role of capillaroscopy. Arthritis Rheum. 2003; 48: 302330.

6. Cutolo M, Pizzorni C, Meroni M, et al. The role of nailfold videocapillaroscopy in Raynaud's phenomenon monitoring and early diagnosis of systemic sclerosis. Reumatismo. 2010; 62: 237-47.

7. Kahaleh MB. Raynaud phenomenon and the vascular disease in scleroderma. Curr Opin Rheumatol. 2004; 16: 718-22.

8. Sulli A, Secchi ME, Pizzorni C, Cutolo M. Scoring the nailfold microvascular changes during the capillaroscopic analysis in systemic sclerosis patients. Ann Rheum Dis. 2008; 67; 885-7.

9. Mugii N, Hasegawa M, Hamaguchi Y, et al. Reduced red blood cell velocity in nailfold capillaries as a sensitive and specific indicator of microcirculation injury in systemic sclerosis. Rheumatology. 2009; 48: 696-703.

10. Murray AK, Moore TL, Manning JB, et al. Non-invasive imaging techniques in the assessment of scleroderma spectrum disorders. Arthritis Rheum. 2009; 61: 1103-11.

11. Ruaro B, Smith V, Sulli A, et al. Methods for the morphological and functional evaluation of microvascular damage in systemic sclerosis. Korean J Intern Med. 2015; 30: 1-5.

12. Cutolo M, Pizzorni C, Sulli A. Capillaroscopy. Best Pract Res Clin Rheumatol. 2005; 19: 437-52.

13. Trombetta AC, Smith V, Pizzorni C, et al. Quantitative alterations of capillary diameter have a predictive value for development of the capillaroscopic systemic sclerosis pattern. J Rheumatol. 2016; 43: 599-606.

14. Kabasakal Y, Elvins DM, Ring EF, McHugh NJ. Quantitative nailfold capillaroscopy findings in a population with connective tissue disease and in normal healthy controls. Ann Rheum Dis. 1996; 55: 507-12.

15. Grassi W, De Angelis R. Capillaroscopy: questions and answers. Clin Rheumatol. 2007; 26: 2009-16.

16. Bernero E, Sulli A, Ferrari G, et al. Prospective capillaroscopy-based study on transition from primary to secondary Raynaud's phenomenon: preliminary results. Reumatismo. 2013; 65: 186-91.

17. Ruaro B, Sulli A, Alessandri E, et al. Coexistence of osteopoikilosis with seronegative spondyloarthritis and Raynaud's phenomenon: first case report with evaluation of the nailfold capillary bed and literature review. Reumatismo. 2012; 64: 335-9.

18. Maricq HR. Wide-field capillary microscopy. Arthris Rheum. 1981; 24: 1159-64.

19. Sulli A, Pizzorni C, Smith V, et al. Timing of transition between capillaroscopic patterns in systemic sclerosis. Arthritis Rheum. 2012; 64: 821-5.

20. Cutolo M, Sulli A, Pizzorni C, Accardo S. Nailfold videocapillaroscopy assessment of microvascular damage in systemic sclerosis. J Rheumatol. 2000; 27: 155-60.

21. Ingegnoli F, Gualtierotti R. A systematic overview on the use and relevance of capillaroscopy in systemic sclerosis. Exp Rev Clin Immunol. 2013; 9: 1091-7.

22. Cutolo M, Pizzorni C, Tuccio M, et al. Nailfold videocapillaroscopic patterns and serum autoantibodies in systemic sclerosis. Rheumatology. 2004; 43: 719-26.

23. Caramaschi P, Canestrini S, Martinelli N, et al. Scleroderma patients nailfold videocapillaroscopic patterns are associated with disease subset and disease severity. Rheumatology. 2007; 46: 1566-9.

24. Sulli A, Ruaro B, Smith V, et al. Progression of nailfold microvascular damage and antinuclear antibody pattern in systemic sclerosis. $\mathbf{J}$ Rheumatol. 2013; 40: 634-9.

25. Sebastiani M, Manfredi A, Colaci M, et al. Capillaroscopic skin ulcer risk index: a new prognostic tool for digital skin ulcer development in systemic sclerosis patients. Arthrit Rheum. 2009; 61: 688-94.

26. Smith V, De Keyser F, Pizzorni C, et al. Nailfold capillaroscopy for day-to-day clinical use: construction of a simple scoring modality as a clinical prognostic index for digital trophic lesions. Ann Rheum Dis. 2011; 70: 180-3.

27. Smith V, Riccieri V, Pizzorni C, et al. Nailfold capillaroscopy for prediction of novel future severe organ involvement in systemic sclerosis. J Rheumatol. 2013; 40: 2023-8.

28. Cutolo M, Herrick AL, Distler O, et al. Nailfold videocapillaroscopic and other clinical 
risk factors for digital ulcers in systemic sclerosis: a multicenter, prospective cohort study. Arthritis Rheumatol. 2016; 68: 2527-39.

29. Riccieri V, Vasile M, Iannace N, et al. Systemic sclerosis patients with and without pulmonary arterial hypertension: a nailfold capillaroscopy study. Rheumatology 2013; 52: 1525-8.

30. Pigatto E, Peluso D, Zanatta E, et al. Evaluation of right ventricular function performed by 3D-echocardiography in scleroderma patients. Reumatismo. 2015; 66: 259-63.

31. Cutolo M. Disease modification in systemic sclerosis. Do integrated approaches offer new challenges? Z Rheumatol. 2013; 72: 326-8.

32. Cutolo M, Sulli A. Therapy: optimized treatment algorithms for digital vasculopathy in SSc. Nature Rev Rheumatol. 2015; 11: 569-71.

33. Catarsi E, Doveri M, Tavoni A. Bosentan and sildenafil: successful treatment in a sclerodermic patient with refractory ulcers. Reumatismo. 2013; 65: 79-81.

34. Soldano S, Montagna P, Brizzolara R, et al. Endothelin receptor antagonists: effects on extracellular matrix synthesis in primary cultures of skin fibroblasts from systemic sclerosis patients. Reumatismo. 2012; 64: 326-34.

35. Casigliani Rabl S, Della Rossa A, Pepe P, et al. Long-term cyclic intravenous iloprost in systemic sclerosis: clinical experience from a single center. Reumatismo. 2012; 64: 158-65.

36. Miniati I, Guiducci S, Conforti ML, et al. Autologous stem cell transplantation improves microcirculation in systemic sclerosis. Ann Rheum Dis. 2009; 68: 94-8.

37. Cutolo M, Ferrone C, Pizzorni C, et al. Peripheral blood perfusion correlates with microvascular abnormalities in systemic sclerosis: a laser-Doppler and nailfold videocapillaroscopy study. J Rheumatol. 2010; 37: 1174-80.

38. Guiducci S, Bellando Randone S, Bruni C, et al. Bosentan fosters microvascular de-remodelling in systemic sclerosis. Clin Rheumatol. 2012; 31: 1723-5.

39. Cutolo M, Zampogna G, Vremis L, et al. Longterm effects of endothelin receptor antagonism on microvascular damage evaluated by nailfold capillaroscopic analysis in systemic sclerosis. J Rheumatol. 2013; 40: 40-5.

40. Cutolo M, Ruaro B, Pizzorni C, et al. Longterm treatment with endothelin receptor antagonist bosentan and iloprost improves fingertip blood perfusion in systemic sclerosis. J Rheumatol. 2014; 41: 881-6.

41. Smith V, Pizzorni C, Riccieri V, et al. Stabilization of microcirculation in patients with early systemic sclerosis with diffuse skin involvement following rituximab treatment: an openlabel study. J Rheumatol. 2016; 43: 995-6.
42. Filaci G, Cutolo M, Scudeletti M, et al. Cyclosporin A and iloprost treatment of systemic sclerosis: clinical results and interleukin-6 serum changes after 12 months of therapy. Rheumatology (Oxford). 1999; 38: 992-6.

43. Aschwanden M, Daikeler T, Jaeger KA, et al. Rapid improvement of nailfold capillaroscopy after intense immunosuppression for systemic sclerosis and mixed connective tissue disease. Ann Rheum Dis. 2008; 67: 1057-9.

44. LeRoy EC, Medsger TA Jr. Criteria for the classification of early systemic sclerosis. J Rheumatol. 2001; 28: 1573-6.

45. Avouac J, Fransen J, Walker UA, et al. Preliminary criteria for the very early diagnosis of systemic sclerosis: results of a Delphi Consensus Study from EULAR Scleroderma Trials and Research Group. Ann Rheum Dis. 2011; 70: 476-81.

46. van den Hoogen F, Khanna D, Fransen J, et al. 2013 classification criteria for systemic sclerosis: an American College of Rheumatology/ European League against Rheumatism collaborative initiative. Arthritis Rheum. 2013; 65: 2737-47.

47. Maverakis E, Patel F, Kronenberg DG, et al. International consensus criteria for the diagnosis of Raynaud's phenomenon. J Autoimmun. 2014; 48: 60-5.

48. Smith V, Beeckman S, Herrick AL, et al. An EULAR study group pilot study on reliability of simple capillaroscopic definitions to describe capillary morphology in rheumatic diseases. Rheumatology. 2016; 55: 883-90.

49. Murray AK, Moore TL, Manning JB, et al. Noninvasive imaging techniques in the assessment of scleroderma spectrum disorders. Arthritis Rheum. 2009; 61: 1103-11.

50. Pizzorni C, Sulli A, Smith V, et al. Capillaroscopy in 2016: new perspectives in systemic sclerosis. Acta Reumatol Port. 2016; 41: 8-14.

51. Roustit M, Cracowski JL. Assessment of endothelial and neurovascular function in human skin microcirculation. Trends Pharmacol Sci. 2013; 34: 373-84.

52. Rosato E, Molinaro I, Rossi C, et al. The combination of laser Doppler perfusion imaging and photoplethysmography is useful in the characterization of scleroderma and primary Raynaud's phenomenon. Scand J Rheumatol. 2011; 40: 292-8.

53. Ruaro B, Sulli A, Smith V, et al. Laser speckle contrast analysis: a new method to evaluate peripheral blood perfusion in systemic sclerosis patients. Ann Rheum Dis. 2014; 73 : 1181-5.

54. Della Rossa A, Cazzato M, d'Ascanio A, et al. Alteration of microcirculation is a hallmark of very early systemic sclerosis patients: a laser 
speckle contrast analysis. Clin Expl Rheumatol. 2013; 31: 109-14.

55. Lambrecht V, Cutolo M, De Keyser F, et al. Reliability of the quantitative assessment of peripheral blood perfusion by laser speckle contrast analysis in a systemic sclerosis cohort. Ann Rheum Dis. 2016; 75: 1263-4.

56. Ruaro B, Sulli A, Pizzorni C, et al. Correlation between skin blood perfusion values and nailfold capillaroscopy scores in systemic sclerosis patients. Microvasc Res. 2016; 105: 119-24.

57. Ruaro B, Sulli A, Smith V, et al. Short-term follow-up of digital ulcers by laser speckle contrast analysis in systemic sclerosis patients. Microvasc Res. 2015; 101: 82-5.

58. Trombetta AC, Pizzorni C, Ruaro B, et al. effects of longterm treatment with bosentan and iloprost on nailfold absolute capillary number, fingertip blood perfusion, and clinical status in systemic sclerosis. J Rheumatol. 2016; 43: 2033-41.

59. Anderson ME, Moore TL, Lunt M, Herrick AL. The 'distal-dorsal difference': a thermographic parameter by which to differentiate between primary and secondary Raynaud's phenomenon. Rheumatology. 2007; 46: 533-8. 